AperTO - Archivio Istituzionale Open Access dell'Università di Torino

Two and three dimensional partition of unity interpolation by product-type functions

This is a pre print version of the following article:

Original Citation:

Availability:

This version is available http://hdl.handle.net/2318/1504213

since 2015-12-03T21:18:14Z

Published version:

DOI:10.12785/amis/090101

Terms of use:

Open Access

Anyone can freely access the full text of works made available as "Open Access". Works made available under a Creative Commons license can be used according to the terms and conditions of said license. Use of all other works requires consent of the right holder (author or publisher) if not exempted from copyright protection by the applicable law. 


\section{(28) \\ UNIVERSITÀ DEGLI STUDI DI TORINO}

This is an author version of the contribution published on:

Questa è la versione dell'autore dell'opera:

[Applied Mathematics \& Information Sciences, volume 9, issue 1, 2015, DOI

10.12785/amis/090101]

ovvero

[R. Cavoretto, volume 9, issue 1, Applied Mathematics \& Information Sciences, 2015, pagg. 1-8]

The definitive version is available at:

La versione definitiva è disponibile alla URL:

[http://www.naturalspublishing.com/Article.asp?ArtclD=7411] 


\title{
Two and three dimensional partition of unity interpolation by product-type functions
}

\author{
Roberto Cavoretto*
}

\begin{abstract}
In this paper we analyze the behavior of product-type radial basis functions (RBFs) and splines, which are used in a partition of unity interpolation scheme as local approximants. In particular, we deal with the case of bivariate and trivariate interpolation on a relatively large number of scattered data points. Thus, we propose the local use of compactly supported $\mathrm{RBF}$ and spline interpolants, which take advantage of being expressible in the multivariate setting as a product of univariate functions. Numerical experiments show good accuracy and stability of the partition of unity method combined with these product-type interpolants, comparing it with the one obtained by replacing compactly supported RBFs and splines with Gaussians.
\end{abstract}

Keywords: multivariate approximation, local interpolation schemes, partition of unity methods, radial basis functions, splines, scattered data.

\section{Introduction}

We consider the problem of interpolating a continuous function $g: \Omega \rightarrow \mathbb{R}$ on a compact domain $\Omega \subset \mathbb{R}^{m}, m=2,3$, defined on a finite set $\mathcal{X}_{N}=\left\{\mathbf{x}_{i}, i=1,2, \ldots, N\right\}$ of data points or nodes, which are situated in $\Omega$. It consists of finding an interpolant $\mathcal{I}: \Omega \rightarrow \mathbb{R}$ such that, given the $\mathbf{x}_{i}$ and the corresponding function values $g_{i}$, the interpolation conditions $\mathcal{I}\left(\mathbf{x}_{i}\right)=g\left(\mathbf{x}_{i}\right)$, $i=1,2, \ldots, N$, are satisfied.

In particular, we are interested in considering the interpolation of large scattered data sets, a problem which has gained much interest in several areas of applied sciences and scientific computing, where the need of having accurate, fast and stable algorithms is often essential (see, e.g, $[17,19,23,24])$. Among the various multivariate approximation techniques, the partition of unity methods such as Shepard's type interpolants turn out to be particularly effective in meshfree or meshless interpolation (see [2, 8, 12, 13, 14, 20, 21, 25, 27, 28]).

In this paper, we propose the use of product-type compactly supported radial basis functions (RBFs) and splines, which are used in a partition of unity interpolation scheme as local approximants. Specifically, here we consider two families of basis functions, known as Wendland functions and Lobachevsky splines, which can be expressed in the multivariate setting as products of univariate functions. The former are well-known in approximation theory and practice, and are usually used as radial functions in the field of multivariate interpolation and approximation (see e.g. [10, 17, 30]). On the other hand, the latter, firstly considered in probability theory $[18,26]$, have successfully been proposed for multivariate scattered data interpolation and integration in $[4,5,6]$ and for landmark-based image registration in $[1,3]$. We remark that both of these families of univariate functions (depending on a shape parameter) are compactly supported, strictly positive definite, and enjoy noteworthy theoretical and computational properties, such as the spline convergence to the Gaussian function. Furthermore, we observe that

\footnotetext{
*Department of Mathematics "G. Peano", University of Torino, via Carlo Alberto 10, I-10123 Torino, Italy. E-mail: roberto.cavoretto@unito.it
} 
the multivariate spline interpolant is noteworthy because it is neither a mesh-based formula nor a radial one, but it asymptotically behaves like a Gaussian interpolant (see [4, 9]). Numerical experiments point out that, for sufficiently regular basis functions such as the product-type radial basis function and spline of smothness $C^{4}$, the partition of unity scheme combined with $\mathrm{RBF}$ and spline interpolants is comparable in accuracy with that obtained by using Gaussian ones, even if they are usually much better conditioned than Gaussians.

The paper is organized as follows. In Section 2 at first we consider the problem of scattered data interpolation by product-type Wendland functions and Lobachevsky splines, recalling their analytic expressions and some properties; then, we describe the partition of unity method, which makes use of product-type RBF and spline interpolants as local approximants. In Section 3 we refer to the corresponding partition of unity algorithms designed for bivariate and trivariate interpolation. Section 4 summarizes several numerical results in order to analyze accuracy and stability of the local approximation scheme combined with compactly supported radial basis function and spline interpolants, also comparing their errors with those of the Gaussian. Finally, Section 5 deals with conclusions and future work.

\section{Partition of unity scheme}

Let us consider a continuous function $g: \Omega \rightarrow \mathbb{R}$ on a compact domain $\Omega \subset \mathbb{R}^{m}, m \geq 1$, a set $\mathcal{X}_{N}=\left\{\mathbf{x}_{i}=\left(x_{1 i}, x_{2 i}, \ldots, x_{m i}\right), i=1,2, \ldots, N\right\} \subset \Omega$ of scattered data points, and the set $\mathcal{G}_{N}=\left\{g\left(\mathbf{x}_{i}\right), i=1,2, \ldots, N\right\}$ of the corresponding function values.

\subsection{Product-type functions}

\subsubsection{Radial basis functions}

In order to construct an interpolation formula generated by compactly supported RBFs, for even $p \geq 0$ we consider the product-type interpolant of the form

$$
F_{p}(\mathbf{x})=\sum_{j=1}^{N} c_{j} \phi_{p j}(\mathbf{x}), \quad \mathbf{x} \in \Omega,
$$

requiring $F_{p}\left(\mathbf{x}_{i}\right)=g\left(\mathbf{x}_{i}\right), i=1,2, \ldots, N$. The interpolant $F_{p}$ is a linear combination of products of univariate shifted and rescaled functions $\zeta_{p}$, called Wendland functions [30], i.e.

$$
\phi_{p j}(\mathbf{x}) \equiv \phi_{p j}(\mathbf{x} ; \delta)=\prod_{h=1}^{m} \zeta_{p}\left(\delta\left(x_{h}-x_{h j}\right)\right),
$$

where, setting $r=\left(x_{h}-x_{h j}\right)$, for $j=1,2, \ldots, N$

$$
\begin{aligned}
& \zeta_{0}(\delta r) \doteq(1-\delta r)_{+}, \\
& \zeta_{2}(\delta r) \doteq(1-\delta r)_{+}^{3}(3 \delta r+1), \\
& \zeta_{4}(\delta r) \doteq(1-\delta r)_{+}^{5}\left(8(\delta r)^{2}+5 \delta r+1\right), \\
& \zeta_{6}(\delta r) \doteq(1-\delta r)_{+}^{7}\left(21(\delta r)^{3}+19(\delta r)^{2}+7 \delta r+1\right),
\end{aligned}
$$

and $\delta \in \mathbb{R}^{+}$is a shape parameter. Note that the support of $\zeta_{p}$ is $[-1 / \delta, 1 / \delta]$. The coefficients $\mathbf{c}=\left\{c_{j}\right\}$ are computed by solving the linear system

$$
A \mathbf{c}=\mathbf{g},
$$

where the interpolation matrix

$$
A=\left\{a_{i j}\right\}=\left\{\phi_{p j}\left(\mathbf{x}_{i}\right)\right\}, \quad i, j=1,2, \ldots, N,
$$


is symmetric and depends on the values of $p$ and $\delta$ in (1). Since these compactly supported RBFs are strictly positive definite, the interpolation matrix $A$ in (2) is positive definite for any set of distinct nodes (see [17]).

Furthermore, since Wendland functions are (univariate) strictly positive definite functions, we can construct multivariate strictly positive definite functions from univariate ones (see, e.g., $[30])$.

Theorem 2.1. Suppose that $\lambda_{1}, \lambda_{2}, \ldots, \lambda_{m}$ are strictly positive definite and integrable functions on $\mathbb{R}$, then

$$
\Lambda(\boldsymbol{x})=\lambda_{1}\left(x_{1}\right) \lambda_{2}\left(x_{2}\right) \cdots \lambda_{m}\left(x_{m}\right), \boldsymbol{x}=\left(x_{1}, x_{2}, \ldots, x_{m}\right) \in \mathbb{R}^{m},
$$

is a strictly positive definite function on $\mathbb{R}^{m}$.

\subsubsection{Spline functions}

For even $n \geq 2$, we construct the product-type spline interpolant of $g$ at the nodes $\mathbf{x}_{i}$ in the form

$$
\tilde{F}_{n}(\mathbf{x})=\sum_{j=1}^{N} \tilde{c}_{j} \tilde{\phi}_{n j}(\mathbf{x}), \quad \mathbf{x} \in \Omega,
$$

requiring $\tilde{F}_{n}\left(\mathbf{x}_{i}\right)=g\left(\mathbf{x}_{i}\right), i=1,2, \ldots, N$. The interpolant $\tilde{F}_{n}$ is a linear combination of products of univariate shifted and rescaled functions $f_{n}^{*}$, known as Lobachevsky splines [4], i.e.

$$
\tilde{\phi}_{n j}(\mathbf{x}) \equiv \tilde{\phi}_{n j}(\mathbf{x} ; \alpha)=\prod_{h=1}^{m} f_{n}^{*}\left(\alpha\left(x_{h}-x_{h j}\right)\right),
$$

where for $j=1,2, \ldots, N$

$$
\begin{aligned}
f_{n}^{*}\left(\alpha\left(x_{h}-x_{h j}\right)\right) & =\sqrt{\frac{n}{3}} \frac{1}{2^{n}(n-1) !} \sum_{k=0}^{n}(-1)^{k}\left(\begin{array}{l}
n \\
k
\end{array}\right) \\
& \times\left[\sqrt{\frac{n}{3}} \alpha\left(x_{h}-x_{h j}\right)+(n-2 k)\right]_{+}^{n-1},
\end{aligned}
$$

or, equivalently,

$$
\begin{aligned}
f_{n}^{*}\left(\alpha\left(x_{h}-x_{h j}\right)\right) & =\sqrt{\frac{n}{3}} \frac{1}{2^{n}(n-1) !} \sum_{k=0}^{\left\lfloor\frac{n}{2}+\frac{1}{2} \sqrt{\frac{n}{3}} \alpha x\right\rfloor}(-1)^{k}\left(\begin{array}{l}
n \\
k
\end{array}\right) \\
& \times\left[\sqrt{\frac{n}{3}} \alpha\left(x_{h}-x_{h j}\right)+(n-2 k)\right]^{n-1}
\end{aligned}
$$

and $\alpha \in \mathbb{R}^{+}$is a shape parameter. Here the support of $f_{n}^{*}$ is given by $[-\sqrt{3 n} / \alpha, \sqrt{3 n} / \alpha]$. The coefficients $\tilde{\mathbf{c}}=\left\{\tilde{c}_{j}\right\}$ are obtained by solving the system of linear equations

$$
\tilde{A} \tilde{\mathbf{c}}=\mathbf{g},
$$

whose interpolation matrix

$$
\tilde{A}=\left\{\tilde{a}_{i j}\right\}=\left\{\tilde{\phi}_{n j}\left(\mathbf{x}_{i}\right)\right\}, \quad i, j=1,2, \ldots, N,
$$

is symmetric and depends on the choice of $n$ and $\alpha$ in (3). As also these splines turn out to be strictly positive definite for any even $n \geq 2$, the interpolation matrix $\tilde{A}$ in (4) is positive definite for any distinct node set. 
From the central limit theorem (see $[18,26]$ ), we remark that the $m$-variate spline converges for $n \rightarrow \infty$ to the $m$-variate Gaussian, i.e.

$$
\lim _{n \rightarrow \infty} \prod_{i=1}^{m} f_{n}^{*}\left(\alpha x_{i}\right)=\frac{1}{(2 \pi)^{m / 2}} \exp \left(\frac{-\alpha^{2}\left(\sum_{i=1}^{m} x_{i}^{2}\right)}{2}\right) .
$$

Hence, these product-type functions asymptotically behave like radial functions, though they are not radial in themselves.

Finally, since also these splines are (univariate) strictly positive definite functions for even $n \geq 2$, from Theorem 2.1 it follows that we can construct multivariate strictly positive definite functions from univariate ones.

\subsection{Interpolation method}

In this subsection we present the partition of unity method. It was firstly suggested in $[7,22]$ in the context of meshfree Galerkin methods for the solution of partial differential equations, but then it became a common tool also used in the field of approximation theory (see [30]).

The basic idea of the partition of unity method is to start with a partition of the open and bounded domain $\Omega \subseteq \mathbb{R}^{m}$ into $d$ subdomains $\Omega_{j}$ such that $\Omega \subseteq \bigcup_{j=1}^{d} \Omega_{j}$ with some mild overlap among the subdomains. Firstly, we take a partition of unity, which consists of a family of compactly supported, non-negative, continuous functions $W_{j}$ with $\operatorname{supp}\left(W_{j}\right) \subseteq \Omega_{j}$ such that

$$
\sum_{j=1}^{d} W_{j}(\mathbf{x})=1
$$

Then, we consider the global approximant of the form

$$
\mathcal{I}(\mathbf{x})=\sum_{j=1}^{d} H_{j}(\mathbf{x}) W_{j}(\mathbf{x}), \quad \mathbf{x} \in \Omega,
$$

where

$$
H_{j}(\mathbf{x})=\sum_{k=1}^{m_{j}} c_{k} \varphi_{k}(\mathbf{x})
$$

is a local interpolant, which is constructed using the $m_{j}$ nodes belonging to the subdomain $\Omega_{j}$ and solving a local interpolation problem. In particular, $H_{j}$ denotes either a RBF interpolant $F_{p j}$ or a spline interpolant $\tilde{F}_{n j}$, whereas $\varphi_{k}$ represents the generic $k$-th basis, $\phi_{p k}$ or $\tilde{\phi}_{n k}$. In fact, we note that if the local approximants satisfy the interpolation conditions at data point $\mathbf{x}_{i}$, i.e.

$$
H_{j}\left(\mathbf{x}_{i}\right)=g\left(\mathbf{x}_{i}\right),
$$

the global approximant also interpolates at this node, i.e.

$$
\mathcal{I}\left(\mathbf{x}_{i}\right)=g\left(\mathbf{x}_{i}\right), \quad i=1,2, \ldots, N .
$$

Then, we give the following definition (see [29]).

Definition 2.1. Let $\Omega \subseteq \mathbb{R}^{m}$ be a bounded set. Let $\{\Omega\}_{j=1}^{d}$ be an open and bounded covering of $\Omega$. This means that all $\Omega_{j}$ are open and bounded and that $\Omega \subseteq \bigcup_{j=1}^{d} \Omega_{j}$. Set $\delta_{j}=\operatorname{diam}\left(\Omega_{j}\right)=$ $\sup _{x, y \in \Omega_{j}}\|\boldsymbol{x}-\boldsymbol{y}\|_{2}$. We call a family of nonnegative functions $\left\{W_{j}\right\}_{j=1}^{d}$ with $W_{j} \in C^{k}\left(\mathbb{R}^{m}\right)$ a $k$-stable partition of unity with respect to the covering $\left\{\Omega_{j}\right\}_{j=1}^{d}$ if

1) $\operatorname{supp}\left(W_{j}\right) \subseteq \Omega_{j}$; 
2) $\sum_{j=1}^{d} W_{j}(\boldsymbol{x}) \equiv 1$ on $\Omega$;

3) for every $\beta \in \mathbb{N}_{0}^{m}$ with $|\beta| \leq k$ there exists a constant $C_{\beta}>0$ such that

$$
\|\left. D^{\beta} W_{j}\right|_{L_{\infty}\left(\Omega_{j}\right)} \leq C_{\beta} / \delta_{j}^{|\beta|}
$$

for all $1 \leq j \leq d$.

In accordance with the statements in [29] we require additional regularity assumptions on the covering $\left\{\Omega_{j}\right\}_{j=1}^{d}$.

Definition 2.2. Suppose that $\Omega \subseteq \mathbb{R}^{m}$ is bounded and $\mathcal{X}_{N}=\left\{\boldsymbol{x}_{i}, i=1,2, \ldots, N\right\} \subseteq \Omega$ are given. An open and bounded covering $\left\{\Omega_{j}\right\}_{j=1}^{d}$ is called regular for $\left(\Omega, \mathcal{X}_{N}\right)$ if the following properties are satisfied:

1) for each $\boldsymbol{x} \in \Omega$, the number of subdomains $\Omega_{j}$ with $\boldsymbol{x} \in \Omega_{j}$ is bounded by a global constant $K$;

2) each subdomain $\Omega_{j}$ satisfies an interior cone condition;

3) the local fill distances $h_{\mathcal{X}_{j}, \Omega_{j}}$ are uniformly bounded by the global fill distance $h_{\mathcal{X}_{N}, \Omega}$, where $\mathcal{X}_{j}=\mathcal{X}_{N} \cap \Omega_{j}$.

Therefore, after defining the space $C_{\nu}^{k}\left(\mathbb{R}^{m}\right)$ of all functions $g \in C^{k}$ whose derivatives of order $|\beta|=k$ satisfy $D^{\beta} g(\mathbf{x})=\mathcal{O}\left(\left.\|\mathbf{x}\|\right|_{2} ^{\nu}\right)$ for $\|\mathbf{x}\|_{2} \rightarrow 0$, we consider the following convergence result (see, e.g., $[17,30])$.

Theorem 2.2. Let $\Omega \subseteq \mathbb{R}^{m}$ be open and bounded and suppose that $\mathcal{X}_{N}=\left\{\boldsymbol{x}_{i}, i=1,2, \ldots, N\right\} \subseteq$ $\Omega$. Let $\varphi \in C_{\nu}^{k}\left(\mathbb{R}^{m}\right)$ be a strictly positive definite function. Let $\left\{\Omega_{j}\right\}_{j=1}^{d}$ be a regular covering for $\left(\Omega, \mathcal{X}_{N}\right)$ and let $\left\{W_{j}\right\}_{j=1}^{d}$ be k-stable for $\left\{\Omega_{j}\right\}_{j=1}^{d}$. Then the error between $g \in \mathcal{N}_{\varphi}(\Omega)$, where $\mathcal{N}_{\varphi}$ is the native space of $\varphi$, and its partition of unity interpolant (5) can be bounded by

$$
\left|D^{\beta} g(\boldsymbol{x})-D^{\beta} \mathcal{I}(\boldsymbol{x})\right| \leq C h_{\mathcal{X}_{N}, \Omega}^{(k+\nu) / 2-|\beta|}|g|_{\mathcal{N}_{\varphi}(\Omega)},
$$

for all $\boldsymbol{x} \in \Omega$ and all $|\beta| \leq k / 2$.

Remark 2.1. We observe that the partition of unity method preserves the local approximation order for the global fit. In fact, we can efficiently compute large product-type interpolants by solving small interpolation problems and then combine them along with the global partition of unity $\left\{W_{j}\right\}_{j=1}^{d}$. This approach allows us to decompose a large problem into many small problems, ensuring at the same time that the accuracy obtained for the local fits is carried over to the global one. In particular, the partition of unity method can be thought as a Shepard's method with higher-order data, since local approximations $H_{j}$ instead of data values $g_{j}$ are used.

\section{Algorithms}

In this section, we describe in detail the partition of unity algorithms used in the interpolation processes, first referring to the bivariate algorithm and then to the trivariate one. They are characterized by the partition of the domain $\Omega$ in square or cube cells, enabling us to use efficient searching procedures. The basic versions of these interpolation algorithms have been proposed and widely tested in $[15,16]$. Here, such algorithms have been modified and efficiently updated to locally apply the product-type interpolants. 


\subsection{Bivariate interpolation}

INPUT:

- $N$, number of data;

- $\mathcal{X}_{N}=\left\{\left(x_{1 i}, x_{2 i}\right), i=1,2, \ldots, N\right\}$, set of data points;

- $\mathcal{G}_{N}=\left\{g_{i}, i=1,2, \ldots, N\right\}$, set of data values;

- $d$, number of subdomains;

- $\mathcal{C}_{d}=\left\{\left(\bar{x}_{1 i}, \bar{x}_{2 i}\right), i=1,2, \ldots, d\right\}$, set of subdomain points;

- $s$, number of evaluation points;

- $\mathcal{E}_{s}=\left\{\left(\tilde{x}_{1 i}, \tilde{x}_{2 i}\right), i=1,2, \ldots, s\right\}$, set of evaluation points.

\section{OUTPUT:}

- $\mathcal{A}_{s}=\left\{\mathcal{I}\left(\tilde{x}_{1 i}, \tilde{x}_{2 i}\right), i=1,2, \ldots, s\right\}$, set of approximated values.

Stage 1. The set $\mathcal{X}_{N}$ of nodes and the set $\mathcal{E}_{s}$ of evaluation points are ordered with respect to the $x_{2}$-axis direction by applying a quicksort $x_{x_{2}}$ procedure.

Stage 2. For each subdomain point $\left(\bar{x}_{1 i}, \bar{x}_{2 i}\right), i=1,2, \ldots, d$, a local circular subdomain is constructed, whose radius depends on the subdomain number $d$, that is

$$
\delta_{\text {subdom }}=\sqrt{\frac{2}{d}} .
$$

This value is suitably chosen, supposing to have a nearly uniform node distribution and assuming that the ratio $n / d \approx 4$.

Stage 3. A double structure of crossed strips is constructed as follows:

i) a first family of $q$ strips, parallel to the $x_{1}$-axis direction, is considered taking

$$
q=\left\lceil\frac{1}{\delta_{\text {subdom }}}\right\rceil,
$$

and a quicksort $x_{1}$ procedure is applied to order the nodes belonging to each strip;

ii) a second family of $q$ strips, parallel to the $x_{2}$-axis direction, is considered.

Note that each of the two strip structures are ordered and numbered from 1 to $q$; moreover, the choice in (7) follows directly from the side length of the domain $\Omega$ (unit square), that here is 1 , and the subdomain radius $\delta_{\text {subdom }}$ in (6).

Stage 4. The unit square is partitioned by a square-based structure consisted of $q^{2}$ squares, whose length of the sides is given by $\delta_{\text {square }} \equiv \delta_{\text {subdom }}$. Then, the following structure is considered:

- the sets $\mathcal{X}_{N}, \mathcal{C}_{d}$ and $\mathcal{E}_{s}$ are partitioned by the square structure into $q^{2}$ subsets $\mathcal{X}_{N_{k}}, \mathcal{C}_{d_{k}}$ and $\mathcal{E}_{s_{k}}, k=1,2, \ldots, q^{2}$,

where $N_{k}, d_{k}$ and $s_{k}$ are the number of points in the $k$-th square.

Stage 5. In order to identify the squares to be examined in the searching procedure, we consider the following two steps: 
(I) since $\delta_{\text {square }} \equiv \delta_{\text {subdom }}$, the ratio between these quantities is denoted by $i^{*}=\delta_{\text {subdom }} / \delta_{\text {square }}$ $=1$. Thus, the number $j^{*}=\left(2 i^{*}+1\right)^{2}$ of squares to be examined for each node is 9 .

(II) for each square $k=[v, w], v, w=1,2, \ldots, q$, a square-based searching procedure is considered, examining the points from the square $\left[v-i^{*}, w-i^{*}\right]$ to the square $\left[v+i^{*}, w+i^{*}\right]$, with the exception of those points close to the boundary of $\Omega$, where we reduce the total number of squares to be examined.

Then, after defining which and how many squares are to be examined, the square-based searching procedure is applied:

- for each subdomain point of $\mathcal{C}_{d_{k}}, k=1,2, \ldots, q^{2}$, to determine all nodes belonging to a subdomain. The number of nodes of the subdomain centred at $\left(\bar{x}_{1 i}, \bar{x}_{2 i}\right)$ is counted and stored in $m_{i}, i=1,2, \ldots, d$;

- for each evaluation point of $\mathcal{E}_{s_{k}}, k=1,2, \ldots, q^{2}$, to find all those belonging to a subdomain of centre $\left(\bar{x}_{1 i}, \bar{x}_{2 i}\right)$ and radius $\delta_{\text {subdom. }}$. The number of subdomains containing the $i$-th evaluation point is counted and stored in $r_{i}, i=1,2, \ldots, s$.

Stage 6. A local interpolant $H_{j}, j=1,2, \ldots, d$, is found for each subdomain point.

Stage 7. A local approximant $H_{j}$ and a weight function $W_{j}, j=1,2, \ldots, d$, is found for each evaluation point.

Stage 8. Applying the global fit (5), one can find approximated values computed at any evaluation point $\left(\tilde{x}_{1}, \tilde{x}_{2}\right) \in \mathcal{E}_{s}$.

\subsection{Trivariate interpolation}

\section{INPUT:}

- $N$, number of data;

- $\mathcal{X}_{N}=\left\{\left(x_{1 i}, x_{2 i}, x_{3 i}\right), i=1,2, \ldots, N\right\}$, set of data points;

- $\mathcal{G}_{N}=\left\{g_{i}, i=1,2, \ldots, N\right\}$, set of data values;

- $d$, number of subdomains;

- $\mathcal{C}_{d}=\left\{\left(\bar{x}_{1 i}, \bar{x}_{2 i}, \bar{x}_{3 i}\right), i=1,2, \ldots, d\right\}$, set of subdomain points;

- $s$, number of evaluation points;

- $\mathcal{E}_{s}=\left\{\left(\tilde{x}_{1 i}, \tilde{x}_{2 i}, \tilde{x}_{3 i}\right), i=1,2, \ldots, s\right\}$, set of evaluation points.

\section{OUTPUT:}

- $\mathcal{A}_{s}=\left\{\mathcal{I}\left(\tilde{x}_{1 i}, \tilde{x}_{2 i}, \tilde{x}_{3 i}\right), i=1,2, \ldots, s\right\}$, set of approximated values.

Stage 1 . The set $\mathcal{X}_{N}$ of nodes and the set $\mathcal{E}_{s}$ of evaluation points are ordered with respect to the $x_{3}$-axis direction by applying a quicksort $x_{3}$ procedure.

Stage 2. For each subdomain point $\left(\bar{x}_{1 i}, \bar{x}_{2 i}, \bar{x}_{3 i}\right), i=1,2, \ldots, d$, a local spherical subdomain is constructed, whose spherical radius depends on the subdomain number $d$, that is

$$
\delta_{\text {subdom }}=\frac{\sqrt{2}}{\sqrt[3]{d}} .
$$

Although other choices $\delta_{\text {subdom }}$ are possible, this value is suitably chosen, supposing to have a nearly uniform node distribution and assuming that the ratio $n / d \approx 8$.

Stage 3. A triple structure of intersecting parallelepipeds is constructed as follows: 
i) a first family of $q$ parallelepipeds, parallel to the $x_{1}$-axis direction, is considered taking

$$
q=\left\lceil\frac{1}{\delta_{\text {subdom }}}\right\rceil,
$$

and a quicksort $x_{1}$ procedure is applied to order the nodes belonging to each parallelepiped;

ii) a second family of $q$ parallelepipeds, parallel to the $x_{2}$-axis direction, is constructed and a quicksort $x_{2}$ procedure is used to order the nodes belonging to each of the resulting parallelepipeds;

iii) a third family of $q$ parallelepipeds, parallel to the $x_{3}$-axis, is considered.

Note that each of the three families of parallelepipeds are ordered and numbered from 1 to $q$; the choice in (9) follows directly from the side length of the domain, i.e. the unit cube, and the subdomain radius $\delta_{\text {subdom }}$ in (8).

Stage 4 . The unit cube is partitioned by a cube-based structure consisted of $q^{3}$ cubes, whose side length is $\delta_{\text {cube }} \equiv \delta_{\text {subdom }}$. Then, the following structure is considered:

- the sets $\mathcal{X}_{N}, \mathcal{C}_{d}$ and $\mathcal{E}_{s}$ are partitioned by the cube structure into $q^{3}$ subsets $\mathcal{X}_{N_{k}}, \mathcal{C}_{d_{k}}$ and $\mathcal{E}_{s_{k}}, k=1,2, \ldots, q^{3}$, where $N_{k}, d_{k}$ and $s_{k}$ are the number of points in the $k$-th cube.

Stage 5. In order to identify the cubes to be examined in the searching procedure, we consider the following two steps:

(I) since $\delta_{\text {cube }} \equiv \delta_{\text {subdom }}$, the ratio between these quantities is denoted by $i^{*}=\delta_{\text {subdom }} / \delta_{\text {cube }}=$ 1. Thus, the number $j^{*}=\left(2 i^{*}+1\right)^{3}$ of cubes to be examined for each node is 27 .

(II) for each cube $k=[u, v, w], u, v, w=1,2, \ldots, q$, a cube-partition searching procedure is considered, examining the points from the cube $\left[u-i^{*}, v-i^{*}, w-i^{*}\right]$ to the cube $\left[u+i^{*}, v+i^{*}, w+i^{*}\right]$, with the exception of those points close to the boundary of $\Omega$, where we reduce the total number of cubes to be examined.

Then, after defining which and how many cubes are to be examined, the cube-partition searching procedure is applied:

- for each subdomain point of $\mathcal{C}_{d_{k}}, k=1,2, \ldots, q^{3}$, to determine all nodes belonging to a subdomain. The number of nodes of the subdomain centred at $\left(\bar{x}_{1 i}, \bar{x}_{2 i}, \bar{x}_{3 i}\right)$ is counted and stored in $m_{i}, i=1,2, \ldots, d$;

- for each evaluation point of $\mathcal{E}_{s_{k}}, k=1,2, \ldots, q^{3}$, in order to find all those belonging to a subdomain of centre $\left(\bar{x}_{1 i}, \bar{x}_{2 i}, \bar{x}_{3 i}\right)$ and radius $\delta_{\text {subdom. }}$. The number of subdomains containing the $i$-th evaluation point is counted and stored in $r_{i}, i=1,2, \ldots, s$.

Stage 6. A local interpolant $H_{j}, j=1,2, \ldots, d$, is found for each subdomain point.

Stage 7. A local approximant $H_{j}$ and a weight function $W_{j}, j=1,2, \ldots, d$, is found for each evaluation point.

Stage 8. Applying the global interpolant (5), one can find approximated values computed at any evaluation point $\left(\tilde{x}_{1}, \tilde{x}_{2}, \tilde{x}_{3}\right) \in \mathcal{E}_{s}$.

\subsection{Complexity}

The algorithms make a repeated use of the quicksort routine along different directions. They require on average a time complexity $\mathcal{O}(M \log M), M$ being the number of nodes to be sorted. In particular, we need a preprocessing phase to build the data structure, whose computational cost is of the order $\mathcal{O}(N \log N)$ for the sorting of all $N$ nodes and $\mathcal{O}(s \log s)$ for the sorting of 
all $s$ evaluation points in Stage 1. Then, to compute the local product-type interpolants, we solve $d$ linear systems of (relatively) small sizes and the computational cost is of order $\mathcal{O}\left(m_{i}^{3}\right)$, $i=1,2, \ldots, d$, for each subdomain, where $m_{i}$ is the number of nodes in the $i$-th subdomain (see Stage 6). Moreover, in Stage 5, 7 and 8 we also have a cost of $r_{k} \cdot \mathcal{O}\left(m_{i}\right), i=1,2, \ldots, d$, $k=1,2, \ldots, s$, for the $k$-th evaluation point of $\mathcal{E}_{s}$.

\section{Numerical results}

In this section we investigate the performances of the partition of unity interpolants obtained by using product-type interpolants as local approximants. In doing so, we focus on accuracy and stability of such interpolation formulas, considering some sets $\mathcal{X}_{N}$ of Halton scattered data points contained in $\Omega=[0,1]^{m} \subset \mathbb{R}^{m}$, for $m=2,3$. They are uniformly distributed random points and generated by using the MATLAB program haltonseq.m [17]. In particular, here we consider some sets of Halton points of size $N=16641,66049$, and $N=35937,274625$, for bivariate and trivariate interpolation, respectively. Note that in this local interpolation scheme we need to solve $d$ linear systems, one for each subdomain $\Omega_{j}, j=1,2, \ldots, d$, of relatively small size $m_{j} \times m_{j}$.

Thus, we analyze the behavior of the partition of unity scheme combined with radial basis function (R) and spline (S) interpolants of smoothness $C^{2}(p=2$ and $n=4)$ and $C^{4}(p=4$ and $n=6$ ), which are denoted by $\mathrm{R} 2, \mathrm{R} 4$ and $\mathrm{S} 2, \mathrm{~S} 4$, respectively. Moreover, for a comparison we also report the results obtained by using the Gaussian $(G)$ as local approximant.

All the results reported below are obtained computing on the following bivariate and trivariate Franke's test functions (see, e.g., [2, 11]), i.e.,

$$
\begin{aligned}
g_{1}\left(x_{1}, x_{2}\right) & =\frac{3}{4} \mathrm{e}^{-\frac{\left(9 x_{1}-2\right)^{2}+\left(9 x_{2}-2\right)^{2}}{4}}+\frac{3}{4} \mathrm{e}^{-\frac{\left(9 x_{1}+1\right)^{2}}{49}-\frac{9 x_{2}+1}{10}} \\
& +\frac{1}{2} \mathrm{e}^{-\frac{\left(9 x_{1}-7\right)^{2}+\left(9 x_{2}-3\right)^{2}}{4}}-\frac{1}{5} \mathrm{e}^{-\left(9 x_{1}-4\right)^{2}-\left(9 x_{2}-7\right)^{2}},
\end{aligned}
$$

and

$$
\begin{aligned}
g_{2}\left(x_{1}, x_{2}, x_{3}\right) & =\frac{3}{4} \mathrm{e}^{-\frac{\left(9 x_{1}-2\right)^{2}+\left(9 x_{2}-2\right)^{2}+\left(9 x_{3}-2\right)^{2}}{4}} \\
& +\frac{3}{4} \mathrm{e}^{-\frac{\left(9 x_{1}+1\right)^{2}}{49}-\frac{9 x_{2}+1}{10}-\frac{9 x_{3}+1}{10}} \\
& +\frac{1}{2} \mathrm{e}^{-\frac{\left(9 x_{1}-7\right)^{2}+\left(9 x_{2}-3\right)^{2}+\left(9 x_{3}-5\right)^{2}}{4}} \\
& -\frac{1}{5} \mathrm{e}^{-\left(9 x_{1}-4\right)^{2}-\left(9 x_{2}-7\right)^{2}-\left(9 x_{3}-5\right)^{2}}
\end{aligned}
$$

the root mean square error (RMSE), whose formula is given by

$$
R M S E=\sqrt{\frac{1}{s} \sum_{i=1}^{s}\left|g\left(\mathbf{x}_{i}\right)-\mathcal{I}\left(\mathbf{x}_{i}\right)\right|^{2}} .
$$

However, in order to have a more complete picture on the local interpolation scheme, we have tested other functions which we here omit for shortness, since accuracy and stability have shown a uniform behavior of the errors.

In Figures 1-2 we show the behavior of compactly supported radial basis function and spline interpolation errors both for $2 \mathrm{D}$ and $3 \mathrm{D}$ cases by varying the value of the shape parameters $\alpha \in[1,10], \delta \in[0.1,1.9]$. Note that there is an exact correspondence between the spline and Gaussian shape parameter $\alpha$, whereas it does not hold by varying $\delta$; hence, though this study turns out to be significant from a numerical standpoint, a direct comparison of $\delta$ and $\alpha$ should be viewed as purely indicative. 
Numerical experiments highlight that the variation of $\alpha$ and $\delta$ may greatly influence the quality of approximation results. Moreover, the behavior of such errors turns out to be more regular and uniform for compactly supported radial basis functions and splines of producttype, whereas it is quite unstable for the Gaussian above all when we take small values of $\alpha$. More precisely, comparing for example spline and Gaussian results, this study points out that the interpolation scheme with product-type interpolants is more stable than the one with the Gaussian. In fact, in the latter case we have interpolation matrices that are very illconditioned, thus producing the fast and sudden error variation shown in Figure 1 for (i) $\alpha \in$ [1,3.9), (ii) $\alpha \in[1,6.3)$, (iii) $\alpha \in[1,2.5)$ and (iv) $\alpha \in[1,3.9)$. Thus, in practice, this error analysis also provides useful information on the conditioning of the different local approximants. Furthermore, analyzing all the tests done, we observe that the product-type functions such as the R4 and S4 (of smoothness $C^{4}$ ) are comparable in accuracy with the Gaussians.

Specifically, we remark that the best level of Gaussian accuracy is reached when $\alpha \in[1,6]$, which is exactly the interval where the Gaussian is unstable. Conversely, though the optimality interval remains the same also for the spline, in that region we have a complete stability; similar considerations, even if depending on the value $\delta$, can also be done for the radial basis function case. Hence, it follows that these tests point out reliability of product-type interpolants also when they are used as local approximants in partition of unity interpolation schemes.

Finally, in Tables 1-2 we report the errors obtained considering optimal values of $\delta$ and $\alpha$, i.e. taking values for which we obtain the smallest RMSEs.

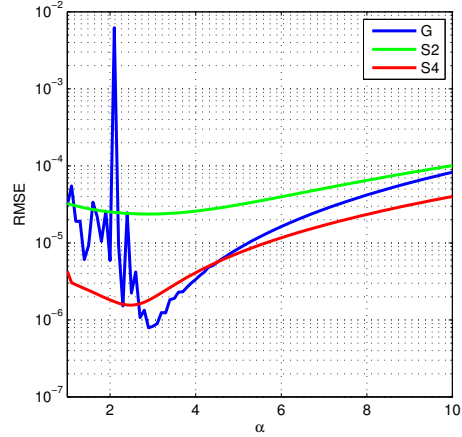

(i) $N=16641-2 \mathrm{D}$

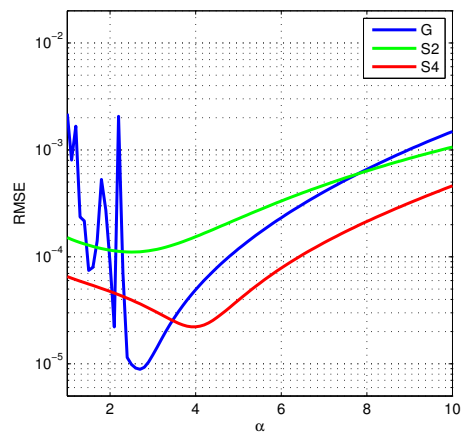

(iii) $N=35937-3 \mathrm{D}$

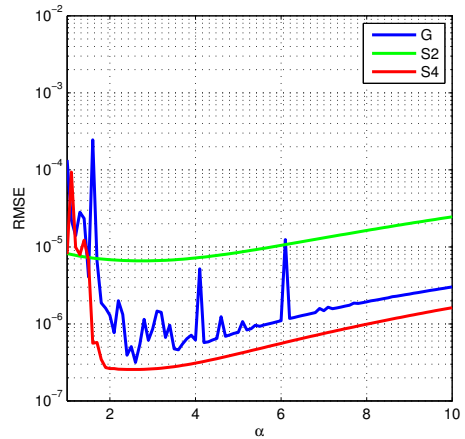

(ii) $N=66049-2 \mathrm{D}$

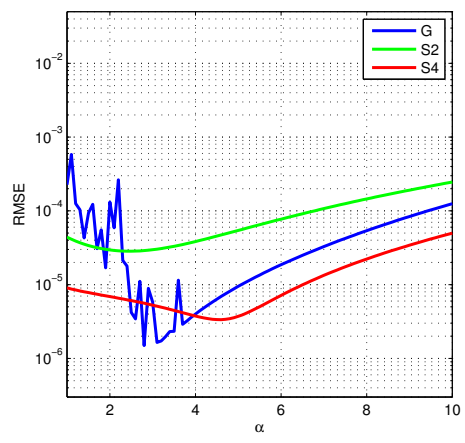

(iv) $N=274625-3 \mathrm{D}$

Figure 1: RMSEs obtained by varying the shape parameters $\alpha$ for bivariate and trivariate Franke's functions. 


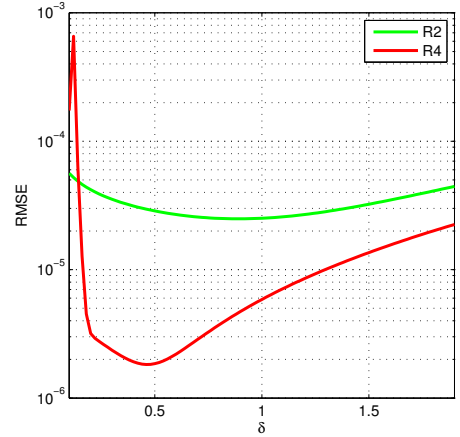

(i) $N=16641-2 \mathrm{D}$

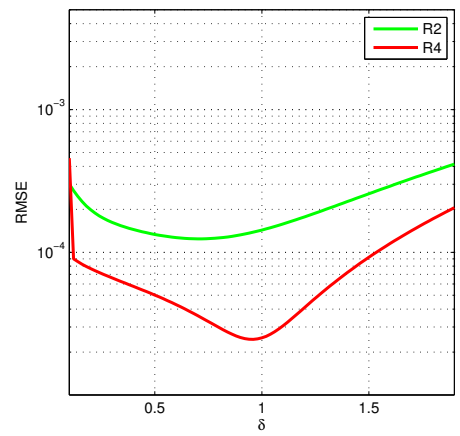

(iii) $N=35937-3 \mathrm{D}$

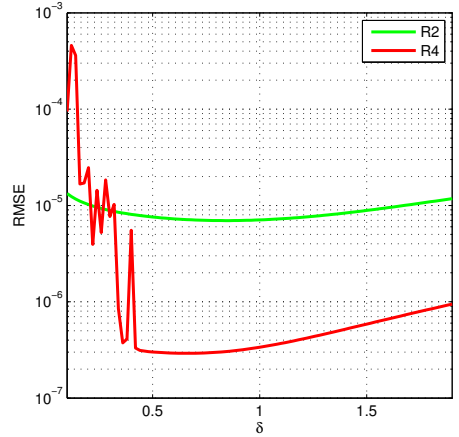

(ii) $N=66049-2 \mathrm{D}$

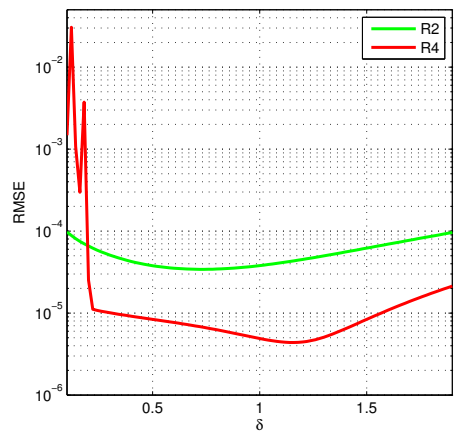

(iv) $N=274625-3 \mathrm{D}$

Figure 2: RMSEs obtained by varying the shape parameters $\delta$ for bivariate and trivariate Franke's functions.

\begin{tabular}{|c|c|c|c|c|}
\hline \multirow{2}{*}{$N$} & \multicolumn{2}{|c|}{16641} & \multicolumn{2}{c|}{66049} \\
\cline { 2 - 5 } & RMSE & $\delta_{\text {opt }}, \alpha_{\text {opt }}$ & RMSE & $\delta_{\text {opt }}, \alpha_{\text {opt }}$ \\
\hline $\mathrm{R} 2$ & $2.4893 \mathrm{E}-5$ & 0.88 & $6.9639 \mathrm{E}-6$ & 0.84 \\
$\mathrm{R} 4$ & $1.8259 \mathrm{E}-6$ & 0.46 & $2.9154 \mathrm{E}-7$ & 0.66 \\
$\mathrm{~S} 2$ & $2.3715 \mathrm{E}-5$ & 2.9 & $6.5901 \mathrm{E}-6$ & 2.7 \\
$\mathrm{~S} 4$ & $1.5553 \mathrm{E}-6$ & 2.5 & $2.5635 \mathrm{E}-7$ & 2.6 \\
$\mathrm{G}$ & $7.9092 \mathrm{E}-7$ & 2.9 & $3.1658 \mathrm{E}-7$ & 2.6 \\
\hline
\end{tabular}

Table 1: RMSEs obtained by using optimal values of $\delta$ and $\alpha$ for $g_{1}$.

\section{Conclusions and future work}

In this paper we studied the performance of product-type interpolants when they are applied as local approximants in a local interpolation scheme such as the partition of unity method. More precisely, we analyzed the behavior of such interpolants in the bivariate and trivariate setting, considering some sets of scattered data points. This study highlighted that these producttype functions work well also in local approaches. Finally, as confirmed by several numerical experiments, they turn out to be more stable than Gaussians and in general, for sufficiently regular basis functions, comparable in accuracy.

As future work, we are going to implement adaptive partition of unity algorithms for scattered data interpolation in high-dimensions, adopting suitable data structures like $k d$-trees and 


\begin{tabular}{|c|c|c|c|c|}
\hline \multirow{2}{*}{$N$} & \multicolumn{2}{|c|}{35937} & \multicolumn{2}{c|}{274625} \\
\cline { 2 - 5 } & $\mathrm{RMSE}$ & $\delta_{\text {opt }}, \alpha_{\text {opt }}$ & $\mathrm{RMSE}$ & $\delta_{\text {opt }}, \alpha_{\text {opt }}$ \\
\hline $\mathrm{R} 2$ & $1.2433 \mathrm{E}-4$ & 0.70 & $3.4168 \mathrm{E}-5$ & 0.74 \\
$\mathrm{R} 4$ & $2.4558 \mathrm{E}-5$ & 0.96 & $4.3705 \mathrm{E}-6$ & 1.16 \\
$\mathrm{~S} 2$ & $1.1088 \mathrm{E}-4$ & 2.5 & $2.8425 \mathrm{E}-5$ & 2.5 \\
$\mathrm{~S} 4$ & $2.2148 \mathrm{E}-5$ & 4.0 & $3.3542 \mathrm{E}-6$ & 4.6 \\
$\mathrm{G}$ & $8.8797 \mathrm{E}-6$ & 2.7 & $1.4928 \mathrm{E}-6$ & 2.8 \\
\hline
\end{tabular}

Table 2: RMSEs obtained by using optimal values of $\delta$ and $\alpha$ for $g_{2}$.

range trees.

\section{Acknowledgement}

The author thanks the financial support of the Department of Mathematics "G. Peano", University of Torino, project "Numerical analysis for life sciences" (2012).

\section{References}

[1] G. Allasia, R. Cavoretto, A. De Rossi, B. Quatember, W. Recheis, M. Mayr, and S. Demertzis, Radial basis functions and splines for landmark-based registration of medical images, In: T.E. Simos et al. (Eds.), Proceedings of the ICNAAM 2010, AIP Conf. Proc. 1281, 716-719 (2010).

[2] G. Allasia, R. Besenghi, R. Cavoretto and A. De Rossi, Scattered and track data interpolation using an efficient strip searching procedure, Appl. Math. Comput. 217, 5949-5966 (2011).

[3] G. Allasia, R. Cavoretto and A. De Rossi, A class of spline functions for landmark-based image registration, Math. Methods Appl. Sci. 35, 923-934 (2012).

[4] G. Allasia, R. Cavoretto and A. De Rossi, Lobachevsky spline functions and interpolation to scattered data, Comput. Appl. Math. 32, 71-87 (2013).

[5] G. Allasia, R. Cavoretto and A. De Rossi, Numerical integration on multivariate scattered data by Lobachevsky splines, Int. J. Comput. Math. 90, 2003-2018 (2013).

[6] G. Allasia, R. Cavoretto and A. De Rossi, Multidimensional Lobachevsky spline integration on scattered data, Appl. Math. Inf. Sci. 8, 145-151 (2014).

[7] I. Babuška and J.M. Melenk, The partition of unity method, Internat. J. Numer. Methods. Engrg. 40, 727-758 (1997).

[8] M.W. Berry and K.S. Minser, Algorithm 798: high-dimensional interpolation using the modified Shepard method, ACM Trans. Math. Software 25, 353-366 (1999).

[9] R. Brinks, On the convergence of derivatives of B-splines to derivatives of the Gaussian functions, Comput. Appl. Math. 27, 79-92 (2008).

[10] M.D. Buhmann, Radial Basis Functions: Theory and Implementation, Cambridge Univ. Press, Cambridge, 2003. 
[11] R. Cavoretto and A. De Rossi, Fast and accurate interpolation of large scattered data sets on the sphere, J. Comput. Appl. Math. 234, 1505-1521 (2010).

[12] R. Cavoretto and A. De Rossi, Spherical interpolation using the partition of unity method: an efficient and flexible algorithm, Appl. Math. Lett. 25, 1251-1256 (2012).

[13] R. Cavoretto, A unified version of efficient partition of unity algorithms for meshless interpolation, In: T.E. Simos et al. (Eds.), Proceedings of the ICNAAM 2012, AIP Conf. Proc. 1479, 1054-1057 (2012).

[14] R. Cavoretto and A. De Rossi, Achieving accuracy and efficiency in spherical modelling of real data, Math. Methods Appl. Sci., (2013). DOI: 10.1002/mma.2906

[15] R. Cavoretto and A. De Rossi, A meshless interpolation algorithm using a cell-based searching procedure, submitted for publication (2013).

[16] R. Cavoretto and A. De Rossi, A trivariate interpolation algorithm using a cube-partition searching procedure, submitted for publication (2013).

[17] G.E. Fasshauer, Meshfree Approximation Methods with MATLAB, World Scientific Publishing, Singapore, 2007.

[18] B.V. Gnedenko, The Theory of Probability, MIR, Moscow, 1976.

[19] A. Iske, Scattered data approximation by positive definite kernel functions, Rend. Sem. Mat. Univ. Pol. Torino 69, 217-246 (2011).

[20] M.A. Iyer, L.T. Watson and M.W. Berry, SHEPPACK: a Fortran 95 package for interpolation using the modified Shepard algorithm, In: R. Menezes et al. (Eds.), Proceedings of the Annual Southeast Conference, 476-481 (2006).

[21] D. Lazzaro and L.B. Montefusco, Radial basis functions for the multivariate interpolation of large scattered data sets, J. Comput. Appl. Math. 140, 521-536 (2002).

[22] J.M. Melenk and I. Babuška, The partition of unity finite element method: basic theory and applications, Comput. Methods. Appl. Mech. Engrg. 139, 289-314 (1996).

[23] V.P. Nguyen, T. Rabczuk, S. Bordas and M. Duflot, Meshless methods: a review and computer implementation aspects, Math. Comput. Simulation 79, 763-813 (2008).

[24] J. Pouderoux, I. Tobor, J.-C. Gonzato and P. Guitton, Adaptive hierarchical RBF interpolation for creating smooth digital elevation models, In: D. Pfoser et al. (Eds.), GIS 2004: Proceedings of the Twelfth ACM International Symposium on Advances in Geographic Information Systems, 232-240 (2004).

[25] R.J. Renka, Multivariate interpolation of large sets of scattered data, ACM Trans. Math. Software 14, 139-148 (1988).

[26] A. Rényi, Calcul des Probabilités, Dunod, Paris, 1966.

[27] E. Sáenz-de-Cabezón, L.J. Hernández, M.T. Rivas, E. García-Ruiz, V. Marco, I. PérezMoreno and F. Javier Sáenz-de-Cabezón, A computer implementation of the partition of the unity procedure and its application to arthropod population dynamics. A case study on the European grape berry moth, Math. Comput. Simulation 82, 2-14 (2011).

[28] W.I. Thacker, J. Zhang, L.T. Watson, J.B. Birch, M.A. Iyer and M.W. Berry, Algorithm 905: SHEPPACK: modified Shepard algorithm for interpolation of scattered multivariate data, ACM Trans. Math. Software 37, Art. 34, 1-20 (2010). 
[29] H. Wendland, Fast evaluation of radial basis functions: methods based on partition of unity, In: C.K. Chui et al. (Eds.), Approximation Theory X: Wavelets, Splines, and Applications, pp. 473-483. Vanderbilt Univ. Press, Nashville, TN (2002)

[30] H. Wendland, Scattered Data Approximation, Cambridge University Press, Cambridge, 2005. 